\title{
25 Research Square \\ Effects Of Kinetic Changes On Neuromuscular Activity Of Adductor Magnus During Bridging Exercises
}

\section{Shutang HE}

First Affiliated Hospital of Sun Yat-sen University

\section{Yashu Li}

First Affiliated Hospital of Sun Yat-sen University

Jiaqi Tan

First Affiliated Hospital of Sun Yat-sen University

Shanshan Zhang

First Affiliated Hospital of Sun Yat-sen University

Chuhuai Wang ( $\nabla$ sumsrehab@163.com )

First Affiliated Hospital of Sun Yat-sen University

\section{Research Article}

Keywords: adductor magnus, bridging exercise, surface electromyography, knee flexion angle.

Posted Date: February 28th, 2022

DOI: https://doi.org/10.21203/rs.3.rs-1369347/v1

License: (c) (i) This work is licensed under a Creative Commons Attribution 4.0 International License. Read Full License 


\section{Abstract}

Background: Most therapeutic exercises can activate the adductor magnus (AM) at a high to a very high level. It is necessary to find an exercise that can facilitate AM at low to moderate levels in the early stage of sports rehabilitation. This study is aimed to investigate changes in AM activation with various knee kinetic positions during bilateral bridging exercise (BBE) and unilateral bridging exercise (UBE).

Method: 20 adults (7 males and 13 females, aged 20-30) volunteered for this study. Surface electromyography (EMG) signals were recorded from $A M$ at four knee flexion angles: $45^{\circ}, 60^{\circ}, 90^{\circ}$, and $120^{\circ}$ when performing UBE and BBE. The obtained raw data were normalized by the corresponding muscle's maximal voluntary isometric contraction (MVIC).

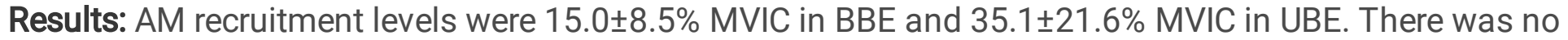
significant difference in the EMG amplitude of $A M$ activity among various knee flexion angles during both UBE and BBE.

Conclusions: BBE and UBE can be utilized as therapeutic exercises to facilitate AM from low to moderate intensity at partly weight-bearing positions.

\section{Background}

The adductor magnus muscle (AM) is a part of hip adductor group muscles, coordinating with pectineus, adductor longus, adductor brevis, and gracilis. It is the largest adductor muscle which takes up $63 \%$ of the mass of the adductor volume and $27 \%$ of the thigh musculature[1, 2]. Also, AM is the third-largest muscle in the lower extremity (smaller than quadriceps femoris and gluteus maximus), which plays a critical role in the terminal stance stage of gait, running, bicycling, and walking up and down stairs [1-3]. However, the possibility of AM injury is lowest in the adductor group muscles strain[4]. On the contrary, AM injury often occurs with the hamstring strain, especially semimembranosus injury[5]. The reason may be that AM consists of two parts, pubofemoral and ischiocondylar portion, the former of which functions as hip adductor muscle and provides pelvic stabilization simultaneously, while the latter originates from the inferior ischial tuberosity, just next to the tendon of the semimembranosus, and is innervated by the common nerve with the hamstring. [2, 6]. Moreover, Benn indicated that the latter was most active during extension and internal rotation, while the former contracted most during extension and adduction[7]. Therefore, the AM also functions as a hip extensor and is called a mini hamstring[6].

The distal posteromedial injury of adductor muscles is associated with AM, which is common in dancer players caused by slow-speed stretching and is one of the main causes of groin pain in soccer players and ice skaters $[5,8,9]$. Moreover, weakness of AM may be a potential factor for hamstring injury[10]. The rehabilitation program for muscle injury begins from maximum protection with no weight-bearing in the acute phase to weight-bearing as tolerated in the subacute stage, then to full-body weight-bearing functional exercises in the chronic phase. However, most of the therapeutic exercises activate AM at a high (41-60\%MVIC) to a very high (>60\%MVIC) level, from mean $47.5 \% \mathrm{MVIC}$ to over $100 \% \mathrm{MVIC}[11,12]$. 
Thus, exercises that can activate $\mathrm{AM}$ at low $(0-20 \% \mathrm{MVIC})$ to moderate $(21-40 \% \mathrm{MVIC})$ levels are necessary for clinical practice.

Bridging exercise, including bilateral bridging exercise (BBE) and unilateral bridging exercise (UBE), is mainly used for the stabilization of the lumbar, trunk and pelvis, and additionally for muscular strength improvement of hip extensor muscles [13]. It's also be considered as a window into both posterior chain function and transverse plane stability. As a partly weight-bearing and functional therapeutic exercise, physiotherapists generally prescribe it as part of the home exercise or rehabilitation program for clients with sports injuries[14]. It may also be used as an effective method for patients with stroke to reinforce hip extensor muscles for gait preparation and to bear low-intensity weight at the early rehabilitation stage [15].

Therefore, the purposes of this study are to determine the following questions:

1. Whether BBE and UBE can be utilized to activate AM? If so, to what extent AM can be activated?

2. Whether the knee flexion angle can affect neuromuscular activation amplitude of AM during BBE and UBE?

\section{Method}

\section{Subjects}

A power analysis was performed with G*Power software ver. 3.1.5 (Franz Faul, University of Kiel, Kiel, Germany) using the results of a pilot study involving 6 participants [16]. The calculation of the sample size was carried out with a power of 0.80 , an alpha level of 0.05 and an effect size of 0.79 . The provided sample size was 16 . Therefore, we recruited 20 healthy volunteers ( 7 males and 13 females, aged 20-30) to participate in the study. All subjects were fully informed of the procedures and purposes of this study and provided written informed consent.

The inclusion criteria were as follows: (1) healthy and physically active subjects, (2) body mass index is between 18.5-24.9 [17], (3) Be willing to sign informed consent. Participants were excluded from the study if they had: (1) a history of lumbar, sacroiliac or lower limb injury within the past year; (2) past or present neurological, musculoskeletal and cardiopulmonary diseases; (3) hip flexor shortness by the Thomas Test and hip abductor tightness by Ober test [18]. (4) lumbar or hip pain when performing bridging exercises. These musculoskeletal examinations of the lower extremity were performed to avoid compensations related to muscle shortness by a principal investigator who is a certified physical therapist. 36 participants were excluded during the clinical screening process, and 20 subjects ultimately participated in this study.

\section{Instrumentation}


Surface electromyography (EMG) was used to record the AM activity during the bridging exercises. EMG data were collected by a four-channel surface electromyogram (United Medical Instruments CO., LTD. Shaoxing, China). Surface electrodes from the same company were made of Ag-Agcl. A manual goniometer was used to measure the different knee angles before performing the bridging exercises.

\section{Testing procedure}

The subjects were informed that they should wear loose and comfortable clothing. To obtain the best estimate of muscle activation, all subjects performed a 5-minute warm-up on the treadmill before the measurement of MVIC[19]. Then, shaved the hair on the belly of AM and cleansed the skin with alcohol. Surface electrodes were attached to the cleansed area with $2 \mathrm{~cm}$ in diameter and fastened with $3 \mathrm{M}$ Transpore $^{\mathrm{TM}}$ medical tape (Micropore Plus, St. Paul, MN). The electrode for the AM was placed mid-way between the medial femoral epicondyle and the pubic tubercle[20]. A common ground electrode was placed on the patella.

MVIC was measured to standardize the action potential of bilateral AM. For the measurement positions and procedures, we followed the method that Kendal et al. proposed [21]. AM contraction was performed for 9 seconds three times, and a 3-minute rest was allowed between each MVIC test to avoid muscular fatigue. For each subject, examiners provided verbal encouragement to help them produce maximum effort during the MVIC.

The subject performed BBE and UBE at $45^{\circ}, 60^{\circ}, 90^{\circ}$ and $120^{\circ}$ knee flexion position respectively. At starting position, their arms crossed over the chest, second toes faced forwards, feet placed a shoulder's width apart and supported on a hard flatbed. When the tester said "Raise your pelvis", the participants raised their pelvises in line with trunk and thigh. When they heard "Hold on", they maintained that position for 9 seconds. When the instruction "Put your hips down" was given, they put their pelvises down and had a rest for 60 seconds. Each type of bridging exercise was performed three times. During these exercises, the knee flexion angle was randomly set at $45^{\circ}, 60^{\circ}, 90^{\circ}$, and $120^{\circ}$, and the position was randomly set with bilateral or unilateral leg support.

\section{Data acquisition}

EMG data of AM were collected from the dominant kicking leg (UBE) and bilateral thigh (BBE) respectively. We performed the motion of each position for 9 seconds and analyzed the muscle activities through measured values for 3 seconds excluding the first 3 seconds and the last 3 seconds to diminish error at the beginning and in the final parts [22]. The raw EMG signals were normalized by MVIC to permit meaningful comparisons among subjects and therefore expressed as a percentage of the MVIC (\% MVIC). When we measured EMG, the sampling rate was $1024 \mathrm{~Hz}$, EMG signals were amplified by 1785 times, the band-pass filter was $20 \sim 450 \mathrm{~Hz}$, and the notch filter was $60 \mathrm{~Hz}$. The collected muscle activity signals were recorded in average electromyography (AEMG) and root mean square (RMS) after full-wave rectification. 


\section{Statistical analysis}

All statistical analyses were performed using SPSS 25.0 (IBM, New York, NY). Descriptive statistics were expressed as a mean and standard deviation. The T-Test was used to compare AM activation amplitude between UBE and BBE. Analyses of variance (ANOVA) was applied to compare the magnitudes of EMG recruitment among four knee flexion angles. If there exists statistical difference, post hoc simple effects tests with Bonferroni adjustments were used to analyze pairwise comparisons between angles. The level of statistical significance was set at 0.05 .

\section{Results}

The basic information and characteristics of twenty subjects were shown in Table 1. AM can be strengthened at low level (AEMG: $16.1 \pm 8.0 \%$ MVIC, RMS: $15.1 \pm 8.3 \% \mathrm{MVIC}$ ) when performing BBE and moderate level (AEMG: $34.7 \pm 17.4 \%$ MVIC, RMS: $35.4 \pm 21.0 \%$ MVIC) when conducting UBE. There exists a significant difference between UBE and BBE at all four knee positions $(P \leq 0.001)$ (Table 2 and Table 3). However, both AEMG and RMS of AM activation revealed no significant differences among four different knee flexion angles during UBE and BBE (Pख0.05) (Table 2 and Table 3).

Table 1. General characteristics of the subjects $\left(M \pm S D^{a}\right)$

\begin{tabular}{|c|c|c|c|c|c|c|}
\hline Gender & $\mathrm{N}$ & Age (years) & Height (m) & Weight (kg) & $\mathrm{BMI}^{\mathrm{b}}\left(\mathrm{kg} / \mathrm{m}^{2}\right)$ & Physical activity (d/wk) \\
\hline male & 7 & $22.6 \pm 4.2$ & $1.73 \pm 0.05$ & $63.3 \pm 8.2$ & $21.2 \pm 2.1$ & $2.0 \pm 1.6$ \\
\hline female & 13 & $21.3 \pm 1.2$ & $1.61 \pm 0.04$ & $51.9 \pm 4.7$ & $20.1 \pm 1.3$ & $1.3 \pm 1.4$ \\
\hline
\end{tabular}

Table 2. Comparison of AM activation (RMS) at four knee flexion angles during UBE and BBE (M $\pm S D)^{a}$ 


\begin{tabular}{|c|c|c|c|c|c|c|c|}
\hline & \multirow[t]{2}{*}{ Group } & \multicolumn{6}{|l|}{$\% \mathrm{MVIC}^{\mathrm{b}}$} \\
\hline & & $45^{\circ}$ & $60^{\circ}$ & $90^{\circ}$ & $120^{\circ}$ & F Value & $P$ \\
\hline \multirow[t]{2}{*}{ RMS } & UBE & $38.7 \pm 27.6$ & $34.4 \pm 20.0$ & $33.2 \pm 17.3$ & $35.3 \pm 18.9$ & .241 & .867 \\
\hline & BBE & $15.7 \pm 9.9$ & $16.1 \pm 10.0$ & $14.7 \pm 7.4$ & $13.8 \pm 5.7$ & .317 & .813 \\
\hline \multicolumn{2}{|c|}{ F Value } & 8.329 & 7.725 & 14.604 & 29.233 & & \\
\hline \multicolumn{2}{|l|}{$P$} & $.001^{*}$ & $.001^{\star}$ & $.000 *$ & $.000 *$ & & \\
\hline \multicolumn{8}{|c|}{$\begin{array}{l}\text { Abbreviations: adductor magnus }=\mathrm{AM} ; \mathrm{UBE}=\text { unilateral bridging; } \mathrm{BBE}=\text { bilateral bridging; }{ }^{\mathrm{a}} \text { mean and } \\
\text { standard deviation; } \mathrm{b} \% \mathrm{MVIC}=\mathrm{RMS} / \mathrm{MVIC}{ }^{\star} 100 \% \text {; }\end{array}$} \\
\hline \multicolumn{8}{|c|}{ *The symbol indicates statistical significance ( $P \leq 0.001)$. } \\
\hline
\end{tabular}

\section{Discussion}

The primary purpose of this study is to identify whether UBE and BBE can be applied to activate AM and if so, to what extent AM can be activated. The second purpose is to determine whether the magnitude of AM recruitment varies among different knee flexion angles. The results show that $A M$ can be strengthened at a low level during BBE and a moderate level during UBE. The intensity for AM recruitment is almost doubled when performing UBE compared with BBE. However, the magnitude of AM activation almost maintains the same with the change of knee flexion angle

Table 3. Comparison of $A M$ activation (AEMG) at four knee flexion angles during UBE and $B B E(M \pm S D)^{a}$

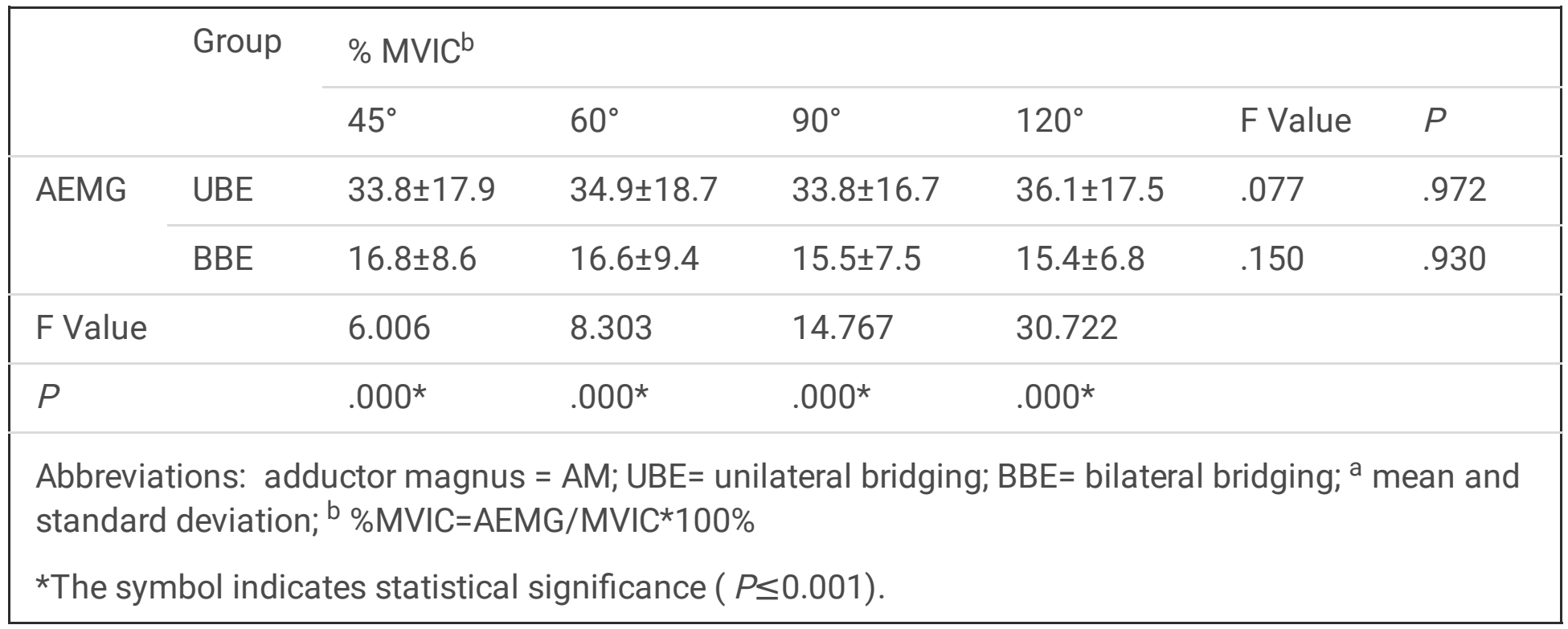

The primary purpose of this study is to identify whether UBE and BBE can be applied to activate AM and if so, to what extent AM can be activated. The second purpose is to determine whether the magnitude of AM recruitment varies among different knee flexion angles. The results show that $A M$ can be strengthened at a low level during BBE and a moderate level during UBE. The intensity for AM recruitment 
is almost doubled when performing UBE compared with BBE. However, the magnitude of AM activation almost maintains the same with the change of knee flexion angle during UBE and BBE.

AM functions as a primary muscle of hip extensors have been demonstrated in previous research [7]. This study showed that there was no significance in AM activation with the increasing knee flexion angles. The reason may be that the AM is a monarticular muscle and the muscular fibre length of which maintains the constant in different knee positions. Moreover, the augmentation of erector spinae muscle activation with the increasing knee flexion angle is also a possible contributing factor for the negative result [23].

The results showed that $A M$ was recruited to a certain extent both during UBE $(35.1 \pm 21.6 \% \mathrm{MVIC})$ and BBE (15.0 $\pm 8.5 \%$ MVIC). However, most of the therapeutic exercises can activate AM at a high (41$60 \% \mathrm{MVIC})$ to a very high ( $>60 \% \mathrm{MVIC}$ ) level. Han-i Ko and colleagues found that AM can be strengthened from $44 \pm 26.5 \% \mathrm{MVIC}$ to $86.2 \pm 46.9 \% \mathrm{MVIC}$ in active prone hip extension with hip adduction or abduction[24]. Another study evaluated the EMG of AM in six clinical examination tests. The authors noted that the AM activities ranged from $47.5 \pm 17.5 \% \mathrm{MVIC}$ to $78.1 \pm 25.9 \% \mathrm{MVIC}[11]$. The rehabilitation program for muscle injury begins from maximum protection with no weight-bearing in the acute phase to weight-bearing as tolerated in the subacute stage, then to full-body weight-bearing functional exercises in the chronic phase. Although the EMG of AM activity did not vary in different knee flexion positions, UBE and BBE still could be applied to strengthen AM at an early stage in sports injury rehabilitation, especially, when the patient is not permitted to perform full weight-bearing tasks.

\section{Conclusion}

Strength training should focus on activating special target muscle groups at a proper level while minimizing the others. BBE and UBE can be utilized as therapeutic exercises to facilitate AM from low to moderate intensity.

\section{Abbreviations}

AM: adductor magnus; BBE: bilateral bridging exercise; UBE: unilateral bridging exercise; EMG: Surface electromyography; MVIC: maximal voluntary isometric contraction; AEMG: average electromyography; RMS: root mean square; ANOVA: Analyses of variance.

\section{Declarations}

\section{Ethics approval and consent to participate}

The study protocol was reviewed and approved by the Human Subjects Ethics Sub-committee of the First Affiliated Hospital, Sun Yat-sen University (Approval number: [2019] 407). All subjects gave written informed consent. All methods were carried out in accordance with the Declaration of Helsinki. 


\section{Consent for publication}

Not applicable

\section{Availability of data and materials}

All data generated or analysed during this study are included in this published article. Competing interests

The authors declare that they have no competing interests.

\section{Funding}

This study was supported by the National Natural Science Foundation of China (81772434) and the Special Fund for Science and Technology of the Industry-University-Research Cooperative Innovation of Guangzhou, China (201704020122).

Authors' contributions: All authors read and approved the final manuscript. SH designed the study and conducted all stages of the study including data collection, analysis, interpretation, and drafting of the manuscript. YL and JT participated in the recruitment and data analysis. SZ revised the manuscript, interpreted the data and managed the trial. CW contributed to study conception and design, data interpretation,

and revising the manuscript.

\section{Acknowledgements}

We thank all volunteers for their availability of time and willingness to participate in the collection procedures.

\section{References}

1. ISHIDA, H., On the muscular composition of lower extremities of apes based on the relative weight. Journal of the Anthropological Society of Nippon, 1972. 80(2): p. 125-142.

2. Takizawa, M., et al., Why adductor magnus muscle is large: the function based on muscle morphology in cadavers. Scand J Med Sci Sports, 2014. 24(1): p. 197-203.

3. Ito, J., et al., Human lower limb muscles: an evaluation of weight and fibre size. Okajimas folia anatomica Japonica, 2003. 80(2.3): p. 47-56.

4. Serner, A., et al., Characteristics of acute groin injuries in the adductor muscles: $A$ detailed MRI study in athletes. Scand J Med Sci Sports, 2018. 28(2): p. 667-676.

5. Askling, C.M., et al., Acute first-time hamstring strains during slow-speed stretching: clinical, magnetic resonance imaging, and recovery characteristics. Am J Sports Med, 2007. 35(10): p. 1716-24. 
6. Broski, S.M., et al., The adductor magnus "mini-hamstring": MRI appearance and potential pitfalls. Skeletal Radiol, 2016. 45(2): p. 213-9.

7. Benn, M.L., et al., Adductor magnus: An EMG investigation into proximal and distal portions and direction specific action. Clin Anat, 2018. 31(4): p. 535-543.

8. Armfield, D.R., et al., Sports-related muscle injury in the lower extremity. Clin Sports Med, 2006. 25(4): p. 803-42.

9. Ibrahim, A., G. Murrell, and P. Knapman, Adductor strain and hip range of movement in male professional soccer players. Journal of Orthopaedic Surgery, 2007. 15(1): p. 46-49.

10. Heynen, M., Hamstring injuries in sprinting. New Studies in Athletics, 2001. 16(3): p. 43-48.

11. Lovell, G.A., P.D. Blanch, and C.J. Barnes, EMG of the hip adductor muscles in six clinical examination tests. Phys Ther Sport, 2012. 13(3): p. 134-40.

12. Hides, J.A., et al., Activation of the hip adductor muscles varies during a simulated weight-bearing task. Phys Ther Sport, 2016. 17: p. 19-23.

13. Kisner, C., L.A. Colby, and J. Borstad, Therapeutic exercise: foundations and techniques. ( 7th ed. ). Philadelphia: F.A. Davis Company 2018: p. pp. 538-539, pp. 758-760.

14. Craig, L., Functional Training Handbook. Wolters Kluwer Health, 2014: p. pp. 71-75.

15. Song, G.B. and J.Y. Heo, The effect of modified bridge exercise on balance ability of stroke patients. J Phys Ther Sci 2015. 27(12): p. 3807-3810.

16. Faul, F., et al., G*Power 3: a flexible statistical power analysis program for the social, behavioral, and biomedical sciences. Behav Res Methods 2007. 39(2): p. 175-191.

17. Cho, S.H., S.H. Kim, and S.Y. Park, Effect of the body mass index and sexual difference on the muscle activity during trunk exercise: a preliminary study. J Exerc Rehabil, 2018. 14(5): p. 778-782.

18. Magee, D., Orthopedic physical assessment. (6th ed.) Philadelphia: WB Saunders, 2014: p. pp. 725727, pp. 729.

19. Merletti, R. and P. Di Torino, Standards for Reporting EMG data. J Electromyogr Kinesiol, 1999. 9(1): p. 3-4.

20. Edward, F.D., et al., Anatomical Guide for the Electromyographer: The Limbs and Trunk. (5th. ed.) Charles C Thomas • Publisher, LTD, 2011: p. pp. 225-229, pp. 247-249, pp. 263-265.

21. Kendall, F.P., E.K. McCreary, and P.G. Provance, Muscles Testing and Function with Posture and Pain. ( 5th ed. ). Lippincott Williams \& Wilkins, 2005: p. pp. 418-419, pp. 426-427, pp. 436-437. 
22. Kim, C.M., et al., The effect of the trunk and gluteus maximus muscle activities according to support surface and hip joint rotation during bridge exercise. Journal of physical therapy science, 2018. 30(7): $p$. 943-947.

23. Hirose, N. and M. Tsuruike, Differences in the electromyographic activity of the hamstring, gluteus maximus, and erector spinae muscles in a variety of kinetic changes. J Strength Cond Res, 2018. 32(12): p. 3357-3363.

24. Ko, H.I., et al., Comparison of hip extensor muscle activity including the adductor magnus during three prone hip extension exercises. Physiother Theory Pract, 2019. 35(5): p. 451-457. 